\title{
Étude méthodologique de la conservation de ressources génétiques de ray-grass anglais (graminée fourragère) par multiplication en pools de populations naturelles
}

\author{
F Balfourier, G Charmet \\ Institut national de la recherche agronomique, \\ station d'amélioration des plantes, 63039 Clermont-Ferrand Cedex, France
}

\begin{abstract}
Résumé - À partir d'une collection de 547 populations naturelles de ray-grass anglais, préalablement étudiées pour des caractères d'intérêt agronomique, 42 populations sont choisies à l'aide d'analyses multivariables et en fonction de critères agronomiques et écogéographiques, afin de constituer 9 pools typés et représentatifs de la diversité observée. Cette méthode de constitution de pools est présentée comme une étape de présélection, permettant la conservation de la diversité et la préparation de matériel génétique original, plus directement utilisable ensuite en sélection. Le choix des critères retenus, pour constituer ces pools, est discuté, puis justifié à l'aide d'analyses discriminantes et d'analyses isoenzymatiques. Enfin, les études expérimentales, à mettre en œuvre pour vérifier la pertinence des regroupements ainsi réalisés, sont proposées.
\end{abstract}

analyse multivariable / ressource génétique / isoenzyme / Lolium perenne / population naturelle

Summary - Methodological study of genetic resources conservation of perennial ryegrass by pooling wild populations. From 547 natural populations of perennial ryegrass previously studied for agronomic characteristics, 42 populations were chosen and regrouped in order to create 9 breeding or pool populations representative of the observed diversity. Agronomic and ecogeographic criteria were taken into account and multivariate analyses were used. This method of pool creation is presented as a pre-breeding process allowing the conservation of diversity and the preparation of genetically original material which can be used directly in breeding programmes. The criteria used in creating the pool populations are discussed and justified with the help of discriminant analyses and isozyme studies. Finally, further experimental studies needed in order to verify the relevance of these groupings are proposed.

multivariate analysis / genetic resource / isoenzyme / Lolium perenne / natural population 


\section{INTRODUCTION}

L'utilisation, à des fins de sélection, de la diversité génétique observée dans une collection de populations naturelles d'une espèce fourragère allogame nécessite des phases préalables d'échantillonnage et de regroupements de ces populations.

En effet, compte tenu de la biologie de ces espèces, il est très contraignant de multiplier, conserver et stocker un grand nombre de populations. Par ailleurs, il est souhaitable de maintenir un maximum de la variabilité collectée. Dans ces circonstances, la constitution d'une «core collection» (Frankel, 1984; Brown, 1989 a et b) apparait être un concept intéressant pour échantillonner des populations dans une vaste collection et définir ainsi une sous-collection ou collection noyau (environ $20 \%$ de l'effectif total) la plus représentative possible de la diversité génétique totale. Cela nécessite de bien penser les critères de choix à utiliser, pour définir ce noyau, sachant que l'utilisation future qui pourra en être faite par le sélectionneur n'est pas toujours connue.

Dans le cas d'une espèce fourragère cultivée comme le ray-grass anglais, les critères à retenir doivent bien évidemment tenir compte des informations issues d'évaluations phénotypiques telles que les caractères morphologiques, physiologiques et d'intérêts agronomiques. Des méthodes d'analyses multivariables, appliquées à ces caractères et prenant en compte les interactions génotype $\times$ milieu, permettent aisément de classer un grand nombre d'accessions (Veronesi et Falcinelli, 1988; Charmet et al, 1990), et s'avèrent être très utiles pour échantillonner des populations parmi un vaste ensemble (Peeters et Martinelli, 1989). Cependant, l'utilisation d'informations écologiques et géographiques issues des données de passeport ou fiches de collecte doit être aussi envisagée. En effet, chez une espèce allogame largement répandue comme le ray-grass anglais, la diversité génétique peut être structurée spatialement, soit du fait de la pression de sélection due aux facteurs environnementaux, soit du fait de phénomènes de dérive ou d'isolement par la distance (Sokal, 1986).

C'est ainsi qu'une «core collection» de ray-grass anglais, issue de la collection française, a pu être récemment définie en combinant 3 critères de choix des populations : leurs ressemblances pour des caractères agronomiques, leur proximité géographique et leur appartenance à une communauté d'habitat (Balfourier et Charmet, 1993).

Toutefois, si la constitution d'une core collection répond bien au souci d'une gestion rationnelle de la variabilité génétique, cela ne permet pas toujours une utilisation rapide de cette variabilité en vue d'une sélection pour la création variétale. En effet, dans le cas d'une espèce comme le ray-grass, la variabilité génétique reste encore très dispersée entre et à l'intérieur des populations qui composent la collection noyau. Dès lors, pour utiliser cette variabilité, une solution consiste à passer par une phase de regroupement de populations par multiplication en panmixie, afin de constituer des pools typés, directement utilisables ensuite dans des programmes de sélection.

L'objectif du présent article est de décrire une méthode de constitution de pools à partir d'une vaste collection de populations naturelles de ray-grass anglais et d'envisager les moyens à mettre en œuvre afin de vérifier la pertinence des regroupements ainsi proposés. 


\section{MATÉRIEL ET MÉTHODES}

\section{Présentation des données utilisées}

Cinq cent quarante-sept populations naturelles de ray-grass anglais ont été collectées sur l'ensemble du territoire continental français, puis évaluées pour des caractères d'intérêts agronomiques dans un réseau multilocal (9 lieux); 10 caractères furent observés en chaque lieu sur une durée de trois ans, sur 30 individus par population étudiés en pépinière de plantes isolées. Les détails concernant l'analyse statistique des données de base, prenant en compte les interactions population $\times$ milieu, ont été décrits par Charmet et al (1990).

Pour le présent article, nous considérerons les moyennes phénotypiques des 10 variables agronomiques mesurées sur les 3 lieux d'étude suivants qui permettaient de déceler une grande part de l'interaction : Clermont-Ferrand, Bourg-Lastic et Le Pin au Haras. La liste de ces variables agronomiques et de leur abréviation est indiquée dans le tableau I.

Tableau I. Variables agronomiques.

\begin{tabular}{llc}
\hline Variable observée & Échelle de notation & Abréviation \\
\hline Sensibilité aux rouilles & $1:$ peu sensible & ROU \\
Sensibilité au froid & $9:$ très sensible & \\
& $1:$ peu sensible & FRO \\
Vigueur au printemps & $9:$ très sensible & \\
& $1:$ peu vigoureux & VIG \\
Aspect estival & $9:$ très vigoureux & \\
& $1:$ peu vigoureux & ETE \\
Redémarrage à l'automne & $9:$ très vigoureux & \\
& $1:$ peu vigoureux & AUT \\
Pérennité en A2 & $9:$ très vigoureux & \\
& $1:$ peu pérenne & PER \\
Alternativité & $9:$ très pérenne & \\
& $1:$ faible & ALT \\
Port & $9:$ forte & \\
& $1:$ dressé & POR \\
Remontaison & $9:$ étalé & REM \\
Épiaison & $1:$ faible & EPI \\
\hline
\end{tabular}

De même, nous utiliserons les informations issues des fiches de site, comprenant notamment des données sur l'habitat d'origine (chemin, prairie, bord de route, friche), le mode d'exploitation (fauche, pâture ou mixte), mais aussi des données géographiques comme la latitude (lat), l'altitude (alt), et climatiques telles que les 
précipitations annuelles (p), l'évapotranspiration (etp), la température moyenne annuelle $(\mathrm{t})$ et les moyennes journalières des températures maxima (tmax) et minima (tmin), respectivement du mois le plus chaud et le plus froid.

\section{Analyse multivariable}

Dans un premier temps, les 29 variables agronomiques furent transformées en variables qualitatives par découpage en 3 classes d'effectif égal. Les variables écogéographiques de type quantitatif subirent la même transformation.

Les 29 variables agronomiques, ainsi transformées, furent ensuite utilisées comme variables actives dans une analyse factorielle des correspondances (AFC), la position de chaque classe de variable sur le plan factoriel 1-2 permettant d'étudier les relations entre ces variables.

Les variables écogéographiques, également rendues qualitatives, furent alors utilisées comme variables additionnelles et projetées sur le même plan factoriel de façon à illustrer les relations entre variables agronomiques et environnementales.

Puis dans un second temps, une classification ascendante hiérarchique, utilisant la méthode UPGMA, a été réalisée sur les 8 premières composantes de l'AFC, de façon à regrouper les populations selon leurs similarités agronomiques. Le dendrogramme obtenu fut ensuite découpé au niveau de 9 classes agronomiques ce qui correspondait à un ratio variance interclasse/variance totale de 0,50 .

Enfin, une étape finale consista à échantillonner des populations parmi un certain nombre de classes agronomiques très typées, de façon à constituer ultérieurement des pools représentatifs de ces classes; 6 classes, dont les barycentres projetés sur le plan factoriel 1-2 étaient assez distants et relativement bien en correspondance avec des variables illustratives très caractéristiques, furent retenues.

Dans chacune de ces 6 classes regroupant des populations aux caractéristiques agronomiques identiques, 4 ou 5 populations furent choisies selon les données de leur site d'origine comme par exemple l'habitat et le mode d'exploitation; 42 populations «mères» ont ainsi été échantillonnées de façon à constituer 9 pools individuels.

\section{Vérification de la robustesse de l'échantillonnage}

Afin de vérifier que notre échantillon n'était pas biaisé, les moyennes de chaque variable agronomique ont été calculées sur chaque pool et comparées à celles des classes d'origine.

Une analyse discriminante a également été réalisée sur les 42 populations, en utilisant les 29 variables agronomiques, de façon à juger de la robustesse et la pertinence de notre choix sur des critères agronomiques.

De même, afin de s'assurer de l'intérêt du critère écologique, une étude électrophorétique, portant sur 6 systèmes enzymatiques (ACP, PGI, GOT, SOD, PRX et IDH) révélant 7 loci polymorphes, a été entreprise sur les populations mères de 3 des pools. Cette étude, utilisant les méthodes décrites par Hayward et Mac Adam (1977), fut réalisée sur environ 150 plantes par populations de façon à calculer les fréquences alléliques des principaux allèles et regarder si ceux-ci permettaient une discrimination des populations en fonction de leurs données écologiques. 


\section{Constitution des pools}

Les 9 pools précédemment définis ont été fabriqués expérimentalement par multiplication en panmixie des populations mères, à raison de 20 plantes par populations, soit $20 \times 5=100$ plantes par pool. De même, les 42 populations mères ont été multipliées individuellement en isolement, à raison d'une soixantaine de plantes par population.

\section{RÉSULTATS}

\section{Relations entre variables agronomiques et écogéographiques}

Le tableau II indique la contribution relative de chaque variable active à l'inertie expliquée par les 2 premiers axes de l'AFC. Ces 2 premiers axes ne représentent que $14 \%$ de l'inertie totale ce qui peut paraître faible. Cependant, d'après Benzecri (1979) et Volle (1985), compte tenu du nombre important de variables utilisées, cette proportion ne donne qu'une vue très biaisée de l'information portée réellement par les axes. L'axe 1 peut être interprété comme un axe de vigueur; en effet, les contributions cumulées les plus fortes sont celles des variables d'aspect estival, de vigueur automnale et pérennité auxquelles sont liées les variables d'alternativité, de remontaison et de sensibilité au froid. Le second axe, quant à lui, est plutôt lié au port, à l'épiaison et à la sensibilité aux rouilles; c'est un axe de «physiologie» de la plante.

La figure 1 montre le plan défini par ces 2 premiers axes. Pour plus de clarté, seules les variables agronomiques du lieu «Bourg-Lastic» (sauf pour l'épiaison observée à Clermont-Ferrand) ont été représentées afin d'illustrer leurs relations avec les variables écogéographiques projetées sur le même plan. Les variables actives figurent en lettres capitales, tandis que les illustratives sont en minuscules. Enfin les barycentres (g1 à g9) des 9 classes agronomiques (issues de la classification) ont été également projetés sur le plan. On observe que l'axe 1 oppose les populations des classes 3 et 7 , plutôt de faibles vigueurs (printemps, été et automne), assez sensibles au froid, alternatives et remontantes (sens négatif de l'axe), aux populations des classes 1,8 et 9 , à l'inverse plus vigoureuses, non alternatives et faiblement remontantes (sens positif de l'axe). L'axe 2 sépare clairement les populations comme celles des classes 4 et 5 , sensibles aux rouilles et à port étalé (sens négatif), des populations les plus tardives, à port dressé et plus résistantes aux rouilles, comme celles des classes 2, 6 et 9 (sens positif).

La figure 2 illustre la localisation des différentes populations sur la carte de France : chaque population est matérialisée par le numéro de sa classe d'appartenance. La figure 3 présente graphiquement les différences entre classes pour certaines variables, l'ensemble des données moyennes pour chaque variable et chaque classe étant disponible sur demande.

Les variables liées à la température ( $t$, tmin, tmax, etp) apparaissent être en étroite correspondance avec les variables de vigueur, de sensibilité au froid, d'alternativité et de remontaison sur l'axe 1. Ainsi les populations des classes 3, 6 et 7 sont plutôt issues des zones les plus chaudes du sud et sud ouest de la France comme le confirme la position de la classe de latitude (lat1). À l'inverse, 
Tableau II. Contribution des variables aux 2 premiers axes de l'AFC.

\begin{tabular}{ccc}
\hline Variables agronomiques & Axe 1 & Axe 2 \\
\hline ROU CL & 0,1 & 7,8 \\
ROU BL & 0,6 & 1,4 \\
ROU LP & 1,4 & 1,0 \\
FRO CL & 1,1 & 3,3 \\
FRO BL & 3,1 & 1,8 \\
FRO LP & 1,5 & 3,4 \\
VIG CL & 1,3 & 5,3 \\
VIG BL & 3,2 & 2,0 \\
VIG LP & 1,2 & 0,3 \\
ETE CL & 5,5 & 1,6 \\
ETE BL & 9,0 & 2,3 \\
ETE LP & 0,9 & 7,1 \\
AUT CL & 4,1 & 5,8 \\
AUT BL & 6,7 & 0,7 \\
AUT LP & 3,3 & 1,2 \\
PER CL & 1,1 & 0,9 \\
PER BL & 5,3 & 0,6 \\
PER LP & 3,6 & 0,6 \\
ALT CL & 9,5 & 2,4 \\
ALT BL & 4,9 & 4,5 \\
ALT LP & 6,9 & 4,0 \\
POR CL & 0,3 & 4,8 \\
POR BL & 0,2 & 5,3 \\
POR LP & 0,5 & 6,1 \\
REM CL & 5,1 & 6,4 \\
REM BL & 6,7 & 1,6 \\
REM LP & 3,5 & 1,0 \\
EPI CL & 4,4 & 8,7 \\
EPI LP & 5,1 & 8,2 \\
\hline
\end{tabular}

les classes 1, 2, 8 et 9 regroupent des populations de sites plus froids de l'est et du nord de la France (lat3). En revanche, la variable p de précipitations apparaît plutôt liée à l'axe 2, en correspondance avec la sensibilité aux rouilles et le port. L'altitude, enfin, apparaît également liée à la sensibilité aux rouilles. Ces résultats sont, dans leur ensemble, tout à fait conformes à ceux décrit par Balfourier et Charmet (1991); d'une manière générale, parmi les facteurs écogéographiques des sites d'origine, les données climatiques semblent plus importantes pour tenter d'expliquer la variabilité.

Cependant, comme on le voit sur la figure 1, quelques variables écologiques telles que l'habitat ou le mode d'exploitation semblent influencer également certains caractères agronomiques. C'est ainsi que les populations issues de prairie apparaissent plus sensibles aux rouilles que celles issues de chemin ou bord de route; de même, 


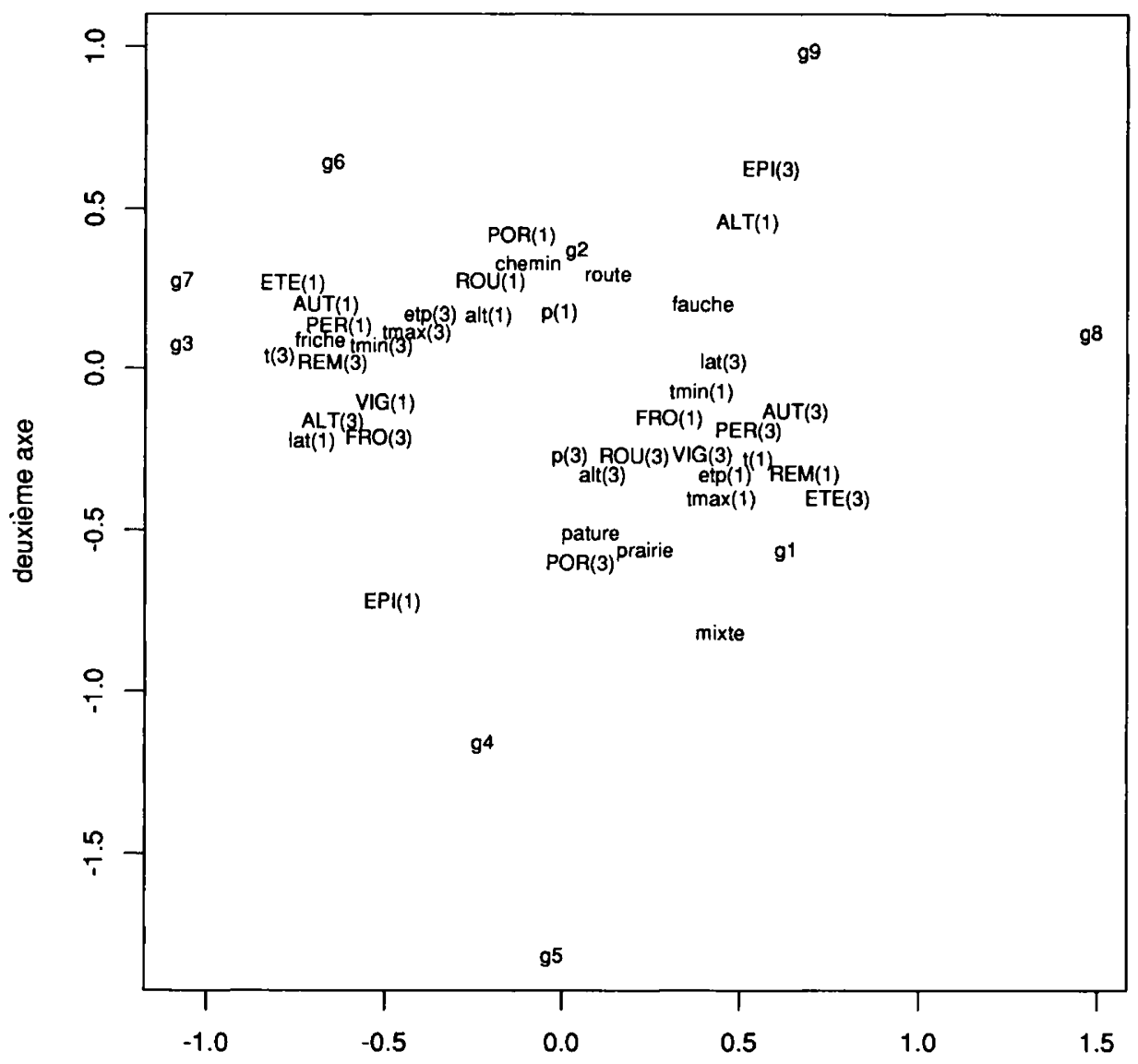

premier axe

Fig 1. Plan 1-2 de l'AFC. Lat : latitude; alt : altitude; $p$ : précipitations annuelles; etp : évapotranspiration; $\mathrm{t}$ : température moyenne annuelle; tmax : moyenne températures maximales; tmin : moyenne températures minimales; $\mathrm{g} 1$ à $\mathrm{g} 9$ : barycentres des classes 1 à 9 ; - pour la signification des autres abréviations, voir tableau $\mathrm{I}$; - le nombre entre parenthèse indique le niveau des classes de variables $(1=$ faible $; 3=$ élevé $)$.

les populations agronomiquement exploitées (pâturées ou fauchées) apparaissent dans l'ensemble plus vigoureuses (côté positif de l'axe 1) que celles non exploitées, par exemple originaires de friches. $Y$ aurait-il ici un phénomène de sélection induit par le mode d'exploitation? 


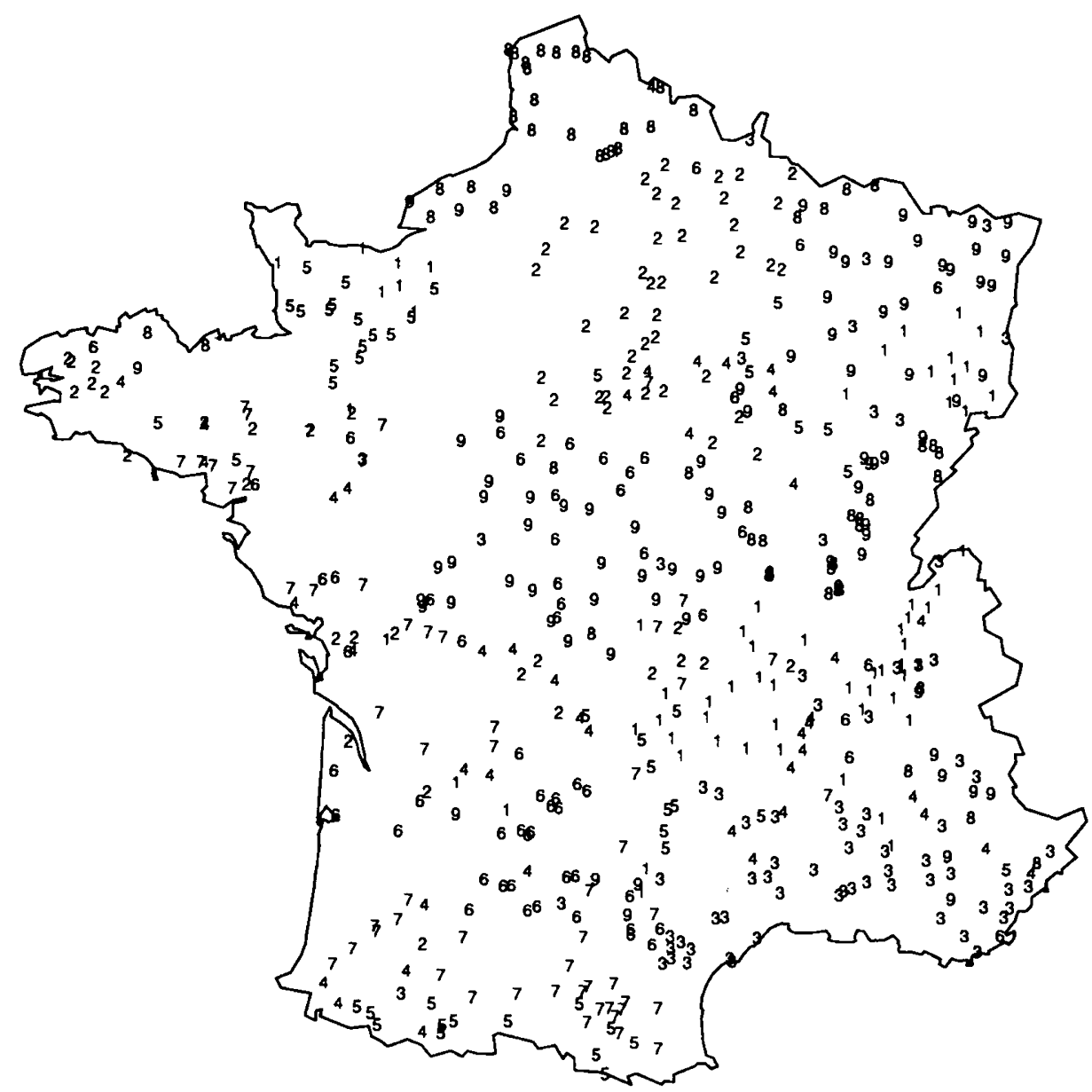

Fig 2. Localisation des populations sur la carte de France. Les populations sont matérialisées par le numéro de leur classe agronomique d'appartenance (1 à 9).

\section{Choix des populations constitutives des pools}

Partant de ces considérations, nous avons fait l'hypothèse que les facteurs environnementaux autres que climatiques intervenaient en tant que facteurs de différenciation naturelle des populations et qu'il était donc nécessaire de prendre en compte aussi ces critères pour le choix des populations destinées à constituer les pools.

Compte tenu de leur bonne représentativité et du positionnement sur le plan 1-2 de leurs caractéristiques agronomiques, les 6 classes agronomiques suivantes ont été choisies : $2,3,5,7,8,9$. Les 42 populations choisies et les 9 pools constitués sont présentés dans le tableau III. Ainsi, chaque population appartenant au même 
vigueur automne $B L$

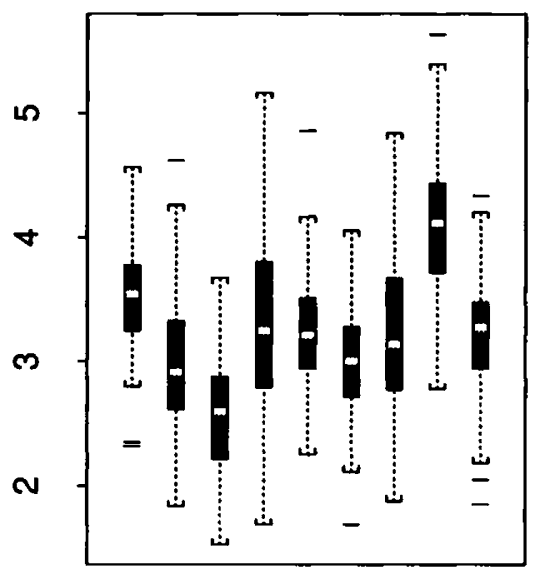

$\begin{array}{lllllllll}1 & 2 & 3 & 4 & 5 & 6 & 7 & 8 & 9\end{array}$

classes agronomiques

port Clermont

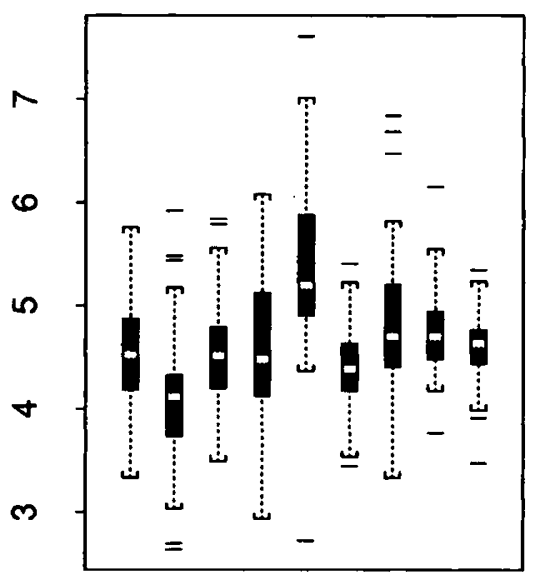

$\begin{array}{lllllllll}1 & 2 & 3 & 4 & 5 & 6 & 7 & 8 & 9\end{array}$

classes agronomiques sensibilité rouilles LP

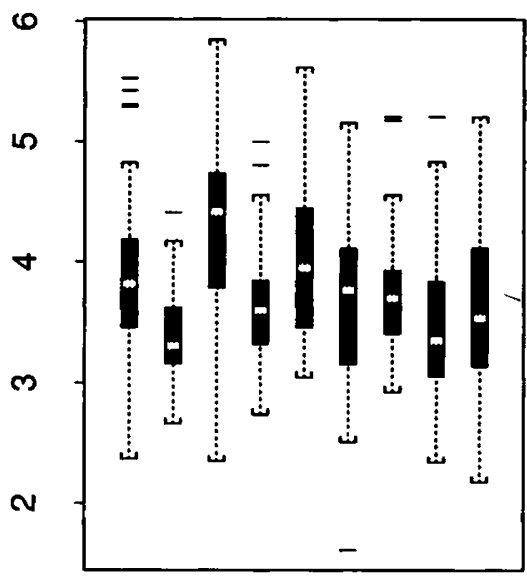

$\begin{array}{lllllllll}1 & 2 & 3 & 4 & 5 & 6 & 7 & 8 & 9\end{array}$

classes agronomiques

date épiaison Clermont

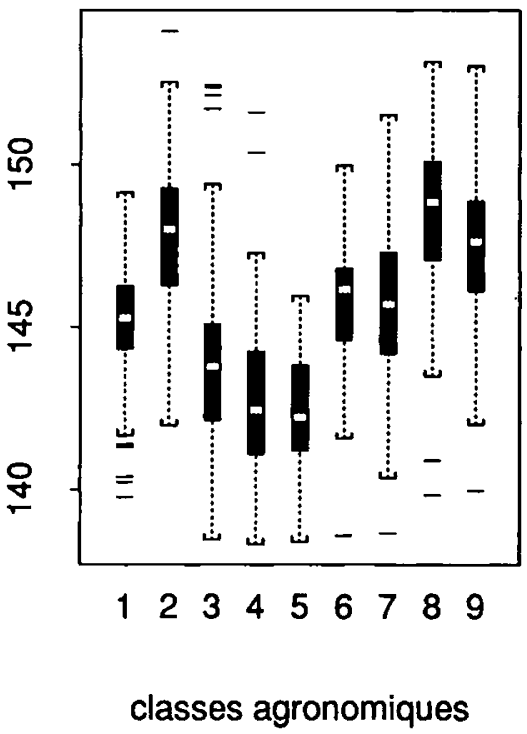

Fig 3. «Boxplots» des 9 classes agronomiques pour des variables observées en différents lieux (BL : Bourg-Lastic; LP : Le Pin au Haras). 
Tableau III. Constitution des pools.

\begin{tabular}{|c|c|c|c|c|}
\hline $\begin{array}{l}\text { Nom de la } \\
\text { population }\end{array}$ & $\begin{array}{c}\text { Classe } \\
\text { agronomique }\end{array}$ & $\begin{array}{c}\text { Habitat } \\
\text { d'origine }\end{array}$ & $\begin{array}{c}\text { Mode } \\
\text { d'exploitation }\end{array}$ & $\begin{array}{c}\text { Pool } \\
\text { d'appartenance }\end{array}$ \\
\hline $\begin{array}{l}\mathrm{CH} 2 \_1 \\
\mathrm{CH} 2 \_2 \\
\mathrm{CH} 2 \_3 \\
\mathrm{CH} 2 \_4\end{array}$ & $\begin{array}{l}2 \\
2 \\
2 \\
2\end{array}$ & Chemin & Non explt & $\mathrm{CH} 2$ \\
\hline $\begin{array}{l}\text { FR3_1 } \\
\text { FR3_2 } \\
\text { FR3_3 } \\
\text { FR3_4 }\end{array}$ & $\begin{array}{l}3 \\
3 \\
3 \\
3\end{array}$ & Friche & Non explt & FR3 \\
\hline $\begin{array}{l}\text { FR7_1 } \\
\text { FR7_2 } \\
\text { FR7_3 } \\
\text { FR7_4 }\end{array}$ & $\begin{array}{l}7 \\
7 \\
7 \\
7\end{array}$ & Friche & \} Non explt & FR7 \\
\hline $\begin{array}{l}\text { PP5_1 } \\
\text { PP5_2 } \\
\text { PP5_3 } \\
\text { PP5_4 } \\
\text { PP5_5 }\end{array}$ & $\begin{array}{l}5 \\
5 \\
5 \\
5 \\
5\end{array}$ & Prairie & Pâturé & PP5 \\
\hline $\begin{array}{l}\text { PF5_1 } \\
\text { PF5_2 } \\
\text { PF5_3 } \\
\text { PF5_4 } \\
\text { PF5_5 }\end{array}$ & $\begin{array}{l}5 \\
5 \\
5 \\
5 \\
5\end{array}$ & Prairie & Fauché & PF5 \\
\hline $\begin{array}{l}\text { PF8_1 } \\
\text { PF8_2 } \\
\text { PF8_3 } \\
\text { PF8_4 } \\
\text { PF8_5 }\end{array}$ & $\begin{array}{l}8 \\
8 \\
8 \\
8 \\
8\end{array}$ & Prairie & Fauché & PF8 \\
\hline $\begin{array}{l}\text { BR8_1 } \\
\text { BR8_2 } \\
\text { BR8_3 } \\
\text { BR8_4 } \\
\text { BR8_5 }\end{array}$ & $\begin{array}{l}8 \\
8 \\
8 \\
8 \\
8\end{array}$ & Bord de route & Fauché & BR8 \\
\hline $\begin{array}{l}\text { PP9_1 } \\
\text { PP9_2 } \\
\text { PP9_3 } \\
\text { PP9_4 } \\
\text { PP9_5 }\end{array}$ & $\begin{array}{l}9 \\
9 \\
9 \\
9 \\
9\end{array}$ & Prairie & Pâturé & PP9 \\
\hline $\begin{array}{l}\text { BR9_1 } \\
\text { BR9_2 } \\
\text { BR9_3 } \\
\text { BR9_4 } \\
\text { BR9_5 }\end{array}$ & $\begin{array}{l}9 \\
9 \\
9 \\
9 \\
9\end{array}$ & Bord de route & Fauché & BR9 \\
\hline
\end{tabular}


pool possède non seulement des caractéristiques agronomiques communes, mais également des caractéristiques de site identiques.

Les comparaisons réalisées sur variables agronomiques entre moyennes des pools et moyennes des classes n'ont pas mis en évidence de différences significatives pour la très grande majorité des variables; on peut donc en conclure que notre échantillon n'est pas biaisé en ce sens qu'il est effectivement représentatif des classes agronomiques échantillonnées.

La figure 4 représente le plan 1-2 de l'analyse discriminante réalisée sur les 42 populations, en utilisant les 29 variables agronomiques. Ce plan représente $85,5 \%$ de l'inertie totale. On observe que les 42 populations sont parfaitement discriminées en 9 pools bien distincts ce qui confirme encore la robustesse de notre méthode d'échantillonnage sur critères agronomiques.

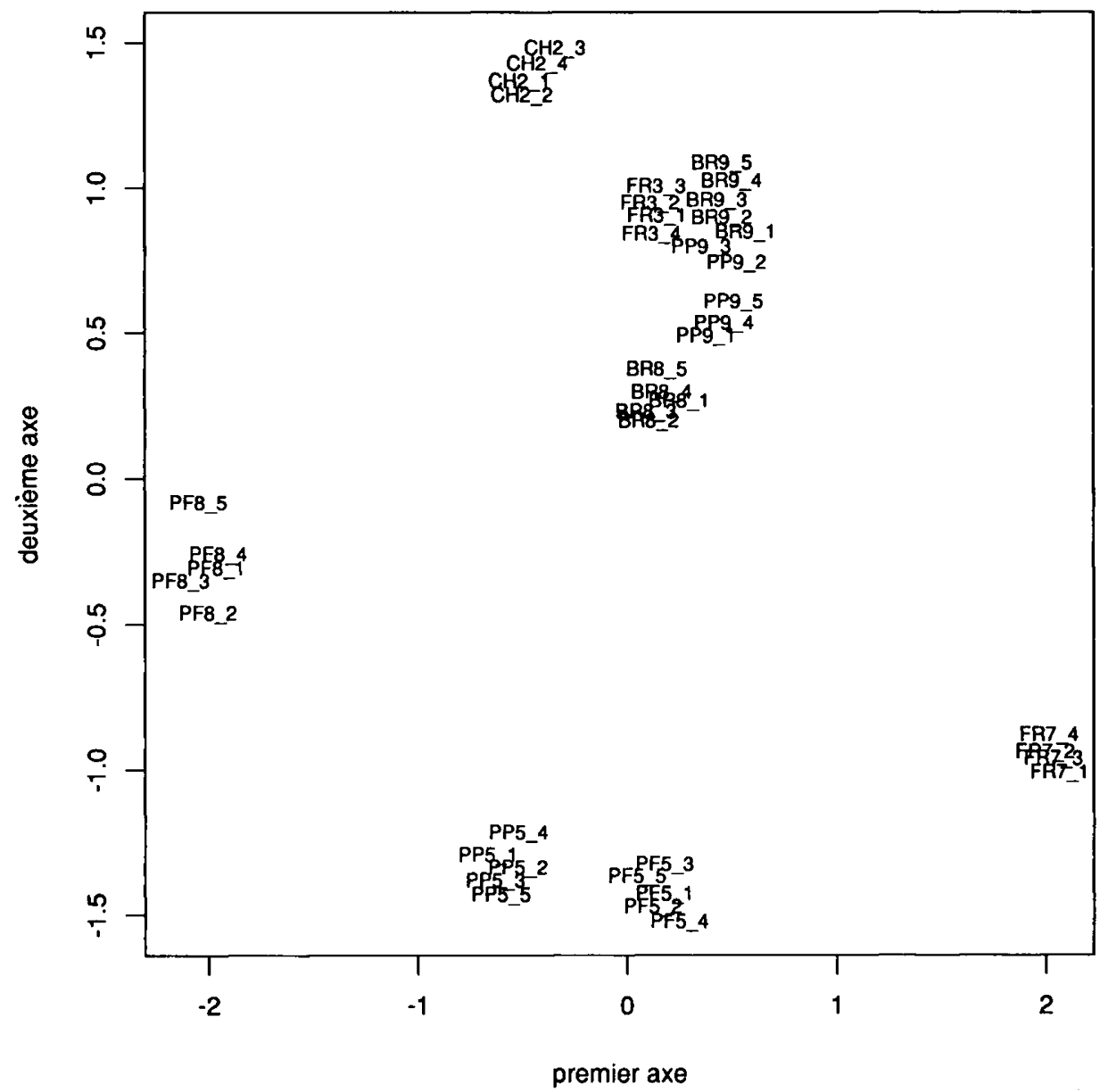

Fig 4. Plan 1-2 de l'AFD sur variables agronomiques. (Pour les abréviations utilisées, voir tableau III). 
Enfin, l'analyse électrophorétique réalisée sur les 14 populations mères des pools PP9, BR9 et CH2 permet de mettre en évidence l'intérêt de retenir, entre autre, l'habitat et le mode d'exploitation comme critères de choix et regroupement des populations en pools; en effet, en considérant les fréquences alléliques d'un seul allèle donné par locus (afin d'éviter la redondance d'information), on peut facilement discriminer ces 14 populations selon leur type d'habitat d'origine; la figure 5 présente le plan factoriel de l'analyse discriminante utilisant 7 fréquences alléliques, soit une par locus polymorphe : les 14 populations sont distinctement discriminées en 3 groupes correspondant aux 3 pools étudiés.

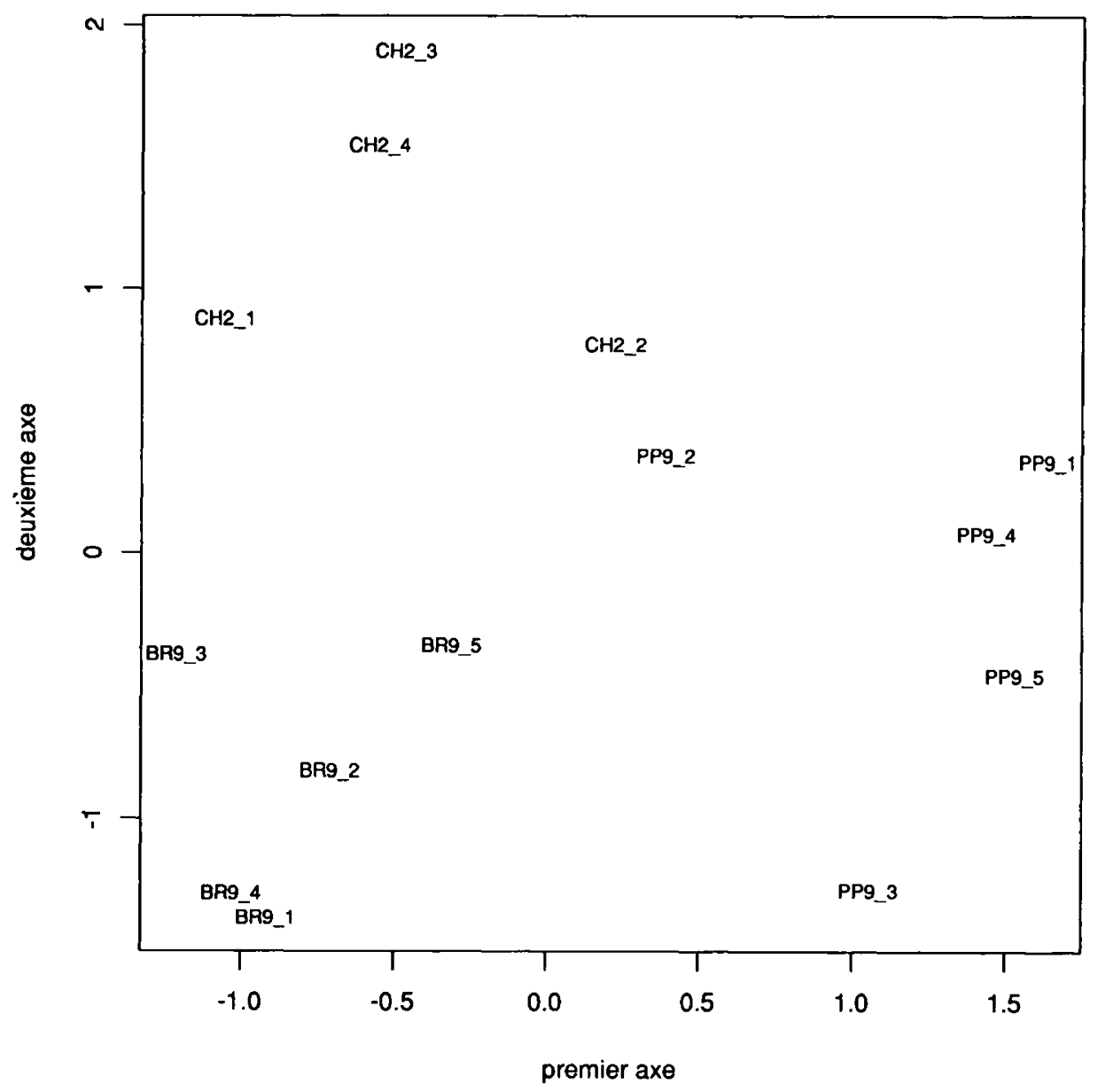

Fig 5. Plan de l'AFD sur fréquences alléliques. (Pour les abréviations utilisées, voir tableau III). 


\section{DISCUSSION}

La méthode expérimentale de constitution de pools présentée ici repose sur le choix d'un certain nombre de critères supposés importants pour expliquer la variabilité génétique observée et donc la conserver.

Ainsi, il semble a priori logique de retenir comme critères ceux issus des évaluations phénotypiques; en effet, la biologie de l'espèce, par exemple, nous impose, dans le cas présent, d'intercroiser les populations selon leur date d'épiaison. Certains caractères morphologiques ou physiologiques, en général assez héritables, doivent aussi être pris en compte (port, alternativité). Quant aux caractères d'intérêts agronomiques (vigueur, sensibilité), bien que moins héritables et soumis aux interactions génotype $\mathrm{x}$ milieu, ils peuvent être pris en compte à condition que leur évaluation soit issue d'observations plurilocales, ce qui est le cas dans la présente étude.

L'utilisation des données de sites, qu'elles soient géographiques (latitude, altitude), climatiques ou écologiques, est en revanche plus délicate. Peteers et al (1990), ont suggéré l'utilisation de telles données pour exploiter la variabilité génétique d'espèces sauvages dont on sait que celle-ci est influencée par les conditions environnementales. D'un autre côté, Weltzein (1989), travaillant sur des populations d'orge, a démontré que des regroupement selon l'origine géographique sont aussi efficaces que ceux effectués sur des similarités morphologiques. Afin d'allier ces 2 types de critères, Gabriel et Sokal (1969) ont proposé des méthodes de classification, sur caractères agromorphologiques, avec contrainte de contiguïté géographique. Cette méthode a été appliquée récemment à la présente collection française et sera publiée prochainement.

L'utilisation de marqueurs biochimiques (isoenzymes) ou moléculaires (RFLP) peut s'avérer aussi une méthode intéressante pour constituer des regroupements selon des critères de distances génétiques. Cependant, on sait qu'il existe peu de corrélation entre distances génétiques et distances géographiques (Lefort-Buson et de Vienne, 1985). En revanche ce sont des outils qui peuvent s'avérer intéressants pour marquer des caractères quantitatifs d'intérêts agronomiques; dans le cas de la collection française de ray-grass, nous n'avons pas utilisé de critère de regroupement portant sur les distances génétiques sachant que, d'après nos observations (Charmet et al, 1993), ces distances entre populations françaises sont très faibles, le maximum de la diversité étant intrapopulations.

A noter que Birouk (1987) a donné un bon exemple d'utilisation de données agronomiques, géographiques, écologiques et isoenzymatiques pour constituer des pools de populations marocaines de luzerne.

Dans le présent article, nous proposons donc d'utiliser a priori non seulement les similarités phénotypiques entre populations, mais également les données écologiques de site. Les analyses discriminantes réalisées tant à partir des variables agronomiques que des fréquences alléliques tendent à nous conforter quant à la justesse des critères retenus et la robustesse des regroupements proposés. En effet, les pools issus des classes 5,8 et 9 ont été constitués de telle sorte que l'on puisse étudier non seulement l'effet «classe agronomique», mais aussi l'effet «habitat» intraclasse. Dans l'analyse discriminante utilisant les variables agronomiques (fig 4), on discrimine parfaitement les populations des pools PP5 et PF5, PF8 et BR8, bien 
que les populations appartiennent respectivement aux même classes 5 et 8 . Enfin, dans l'analyse avec les fréquences alléliques (fig 5), les populations des pools PP9 et BR9 sont également bien discriminées selon l'habitat, alors qu'elles proviennent de la même classe agronomique.

\section{CONCLUSION}

Les avantages et inconvénients concernant l'intérêt de la création de pools pour la conservation et l'utilisation des ressources génétiques ont été largement développés par Guy et al (1989).

La méthode de création de pools décrite ici apparaît comme une stratégie de présélection permettant d'allier la conservation "dynamique» de la diversité à sa mobilisation en vue de constituer du matériel génétique plus directement valorisable.

Cette méthode n'a d'intérêts que si elle permet d'obtenir du matériel génétique original, bien caractéristique et facilement utilisable en sélection. Cela nécessite une bonne connaissance du matériel génétique de base, tant sur le plan de ses caractéristiques agronomiques intrinsèques que sur ses origines écogéographiques.

En fait, l'intérêt du regroupement en pools, par rapport à une conservation de populations isolément, réside dans l'espoir d'obtenir l'expression d'une plus grande variabilité par le jeu des recombinaisons génétiques entre populations du même pool. Le risque est en revanche de détruire les balances internes pouvant exister à i'intérieur des populations.

C'est pourquoi, une fois les pools constitués par multiplication de leurs populations mères en panmixie, il conviendra de vérifier expérimentalement la pertinence de nos regroupements, notamment grâce à :

- l'étude de la conservation, dans les pools, des fréquences alléliques et de la structure génétique présentes dans leurs populations mères;

- l'étude de la représentativité et la stabilité des pools pour des critères multivariables;

- enfin, l'étude de l'influence des regroupements effectués sur les paramètres génétiques et la réponse à la sélection.

Ces 3 études sont actuellement en cours de réalisation; l'ensemble des résultats devrait être publié prochainement.

\section{REMERCIEMENTS}

Les auteurs souhaitent remercier les sélectionneurs des établissements privés membres de l'Association des créateurs de variétés fourragères. Ce travail a été réalisé pour partie grâce à des contrats de branche avec le MAF, ainsi qu'à l'aide d'une action FRT (n॰88 R 0704). 


\section{RÉFÉRENCES}

Balfourier F, Charmet G (1991) Relationships between agronomic characters and ecogeographical factors in a collection of French perennial ryegrass populations. agronomie 11, 645-657

Balfourier F, Charmet G (1993) Use of ecogeographical factors for sampling a core collection of perennial ryegrass. In: Proc XVII Inter Grassland Congress Palmerston North, NZ 228-230

Benzecri JP (1979) Sur le calcul des taux d'inertie dans l'analyse d'un questionnaire. Cah Anal Données 4 (3), 377-378

Birouk A (1987) Les ressources phytogénétiques au Maroc : analyse de la variabilité génétique d'une espèce fourragère : la luzerne (Medicago sativa). Thèse Université Paris-Sud

Brown AHD (1989a) The case for core collection. In: The use of plant genetic resources (Brown AHD, Frankel OH, Marshall DR, Williams JT, eds). Cambridge University Press, Cambridge, Vol 3, 136-156

Brown AHD (1989b) Core collection: a practical approach to genetic resources management. Genome 31, 818-824

Charmet G, Balfourier F, Bion A (1990) Agronomic evaluation of a collection of French perennial ryegrass populations: multivariate classification using genotype $\mathrm{x}$ environment interactions. Agronomie 10, 807-823

Charmet G, Balfourier F, Ravel C (1993) Isoenzyme polymorphism and geographic differenciation in a collection of French perennial ryegrass populations. Genet Res Crop Evol 40, 77-89

Frankel OH (1984) Genetic perspectives of germplasm conservation. In: Genetic manipulation: impact of man and society (Arbez M, Illimense WK, Peacock WJ, Starlinger P, eds). Cambridge University Press, Cambridge, 161-170

Gabriel KR, Sokal RR (1969) A new statistical approach to geographic variation analysis. Syst Zool 18, 159-270

Guy P, Ghesquière M, Charmet G, Prospéri JP (1989) Pooling accessions: Advantages and disadvantages. In: Rapport du groupe de travail sur les fourrages (IBPGR). Montpellier, 9-12 janvier 1989, 35-49

Hayward MD, Mac Adam NJ (1977) Isozyme polymorphism as a measure of distinctiveness and stability in cultivars of Lolium perenne. $Z$ Pflanzenzucht 79 , 59-68

Lefort-Buson M, De Vienne D (1985) Les distances génétiques. INRA Publications, Versailles, France

Peeters JP, Martinelli JA (1989) Hierarchical clustering analysis as a tool to manage variation in germplasm collection. Theor Appl Genet 78, 42-48

Peeters JP, Wilkes HG, Galwey NW (1990) The use of ecogeographical data in the exploitation of variation from gene bank. Theor Appl Genet 80, 110-112

Sokal RR (1986) Spatial data analysis and historical process. In: Data analysis and informatics IV. Elsevier, Amsterdam 
Veronesi F, Falcinelli M (1988) Evaluation of an Italian germplasm collection of Festuca arundinacea-Schreb through a multivariate analysis. Euphytica 38, 211-220 Volle (1985) Analyse des données. Economica, Paris

Weltzein E (1989) Differentiation among barley landrace populations from the Near East. Euphytica 43, 29-39 